\title{
Competition between strains of Borrelia afzelii in the host tissues and consequences for transmission to ticks
}

\author{
Dolores Genné $\mathbb{D}^{1} \cdot$ Marika Rossel $^{1} \cdot$ Anouk Sarr $^{1} \cdot$ Florian Battilotti $^{1} \cdot$ Olivier Rais ${ }^{2} \cdot$ Ryan O. M. Rego $\mathbb{C}^{3,4}$ • \\ Maarten J. Voordouw (1D ${ }^{1,5}$
}

Received: 1 July 2020 / Revised: 26 January 2021 / Accepted: 11 February 2021 / Published online: 3 March 2021

(c) The Author(s) 2021. This article is published with open access

\begin{abstract}
Pathogen species often consist of genetically distinct strains, which can establish mixed infections or coinfections in the host. In coinfections, interactions between pathogen strains can have important consequences for their transmission success. We used the tick-borne bacterium Borrelia afzelii, which is the most common cause of Lyme disease in Europe, as a model multi-strain pathogen to investigate the relationship between coinfection, competition between strains, and strain-specific transmission success. Mus musculus mice were infected with one or two strains of B. afzelii, strain transmission success was measured by feeding ticks on mice, and the distribution of each strain in six different mouse organs and the ticks was measured using qPCR. Coinfection and competition reduced the tissue infection prevalence of both strains and changed their bacterial abundance in some tissues. Coinfection and competition also reduced the transmission success of the B. afzelii strains from the infected hosts to feeding ticks. The ability of the B. afzelii strains to establish infection in the host tissues was strongly correlated with their transmission success to the tick vector. Our study demonstrates that coinfection and competition between pathogen strains inside the host tissues can have major consequences for their transmission success.
\end{abstract}

\section{Introduction}

Most pathogen species are composed of different strains [1], which can vary in traits, such as infection intensity, virulence, and transmission [2-4]. This pathogen strain diversity

Supplementary information The online version contains supplementary material available at https://doi.org/10.1038/s41396021-00939-5.

$\triangle$ Dolores Genné

dolores.genne@unine.ch

1 Laboratory of Ecology and Evolution of Parasites, Institute of Biology, University of Neuchâtel, Neuchâtel, Switzerland

2 Laboratory of Ecology and Epidemiology of Parasites, Institute of Biology, University of Neuchâtel, Neuchâtel, Switzerland

3 Biology Centre, Institute of Parasitology, Czech Academy of Sciences, České Budějovice, Czechia

4 Faculty of Science, University of South Bohemia, České Budějovice, Czechia

5 Department of Veterinary Microbiology, Western College of Veterinary Medicine, University of Saskatchewan, Saskatoon, Canada can result in mixed infections or coinfections when hosts are infected with multiple strains $[1,2,5]$. Mixed infections can alter the fitness of the co-infecting strains compared to when they are alone in the host. However, the outcome of interactions between strains is highly variable and can range from facilitation to competition [5-7]. Furthermore, the performances of strains when they are alone in their host, are often not predictive of their performances when they are in a mixed infection $[5,8]$. In summary, in systems where coinfections are common, interactions between strains may have important consequences for their transmission and hence the strain composition of the pathogen population.

Vector-borne pathogens are interesting systems for the study of mixed infections because pathogen strains can interact in both the vertebrate host [9-13] and the arthropod vector [14-17]. However, few studies have shown a direct relationship between strain-specific abundance in the tissues of co-infected hosts and strain-specific host-to-vector transmission success $[3,18]$. In a rodent malaria system, the strain of Plasmodium chabaudi that established the highest abundance in the mouse blood was also the strain that had the highest mouse-to-mosquito transmission success [3]. Other vector-borne pathogens are found in tissues such as the skin, where the interactions between strains may 
be very different compared to a well-mixed liquid tissue such as the blood. Thus, studies of other systems will enhance our understanding of how inter-strain interactions in the vertebrate host shape strain-specific transmission success to the arthropod vector.

Our research group uses the tick-borne pathogen, Borrelia afzelii, as a model system to study interactions between strains in the rodent host and the tick vector. This spirochete bacterium belongs to the Borrelia burgdorferi sensu lato (sl) genospecies complex and is one of the most common causes of Lyme disease in Europe. B. afzelii is transmitted by the hard tick Ixodes ricinus and uses small mammals (e.g. rodents and shrews) as reservoir hosts [19]. I. ricinus consists of three different stages, larva, nymph, and adult, and each stage requires a blood meal to moult to the next stage, or to produce eggs in the case of adult females. Vertical transmission of spirochetes is rare [20], and larval $I$. ricinus ticks acquire $B$. afzelii during their first blood meal from an infected host. The skin, rather than the blood, is believed to be the critical organ for transmission of B. afzelii to feeding I. ricinus ticks [21-23].

B. afzelii contains dozens of genetically distinct strains $[24,25]$ and strain diversity is high at small spatial scales [9, 11, 26-30]. Both small mammal hosts $[9,11,31]$ and I. ricinus ticks $[26,27]$ can be co-infected with multiple strains of B. afzelii. Previous studies on B. burgdorferi sl have found that coinfection in the vertebrate host results in competitive interactions that reduce the strain-specific abundance in host tissues [11] and the strain-specific hostto-tick transmission [14, 15, 32]. Suggested mechanisms of inter-strain competition include resource competition over limited host resources (e.g., nutrients, space) and apparent competition mediated by the host immune system [6, 7]. Surprisingly, there are no controlled studies for any $B$. burgdorferi sl genospecies that have investigated whether the effects of coinfection on strain-specific abundance in the host tissues influence strain-specific host-to-tick transmission success.

In this study, we used two strains of B. afzelii to investigate their interactions in a suitable rodent host, the laboratory mouse Mus musculus. For mice infected with single strains and for co-infected mice, we quantified the strain-specific presence and abundance in different organs and the strain-specific host-to-tick transmission success. We predict (1) that competition between strains will reduce the strain-specific presence and abundance in tissues of coinfected mice compared to mice with single strain infections; (2) that strains will exhibit preferences for different organs (strain-specific tissue tropism) and that the strength of inter-strain competition will differ among organs; (3) that competition between strains will reduce their transmission success from infected mice to feeding ticks; and (4) that the strain-specific presence and abundance in the host tissues will determine the strain-specific host-to-tick transmission success.

\section{Materials and methods}

\section{Mice, ticks, and Borrelia afzelii strains}

Forty female, 5-week-old, specific pathogen-free Mus musculus $\mathrm{BALB} / \mathrm{c}$ mice were used in this study. We used $\mathrm{BALB} / \mathrm{c}$ mice because we have a history of successful experimental infections with this mouse strain [33-35]. The I. ricinus ticks came from the laboratory colony that has been maintained at the University of Neuchâtel since 1978. We used B. afzelii strains NE4049 and Fin-Jyv-A3 in this study because we have a history of successful experimental infections with these two strains [33, 34, 36, 37]. Strain NE4049 was isolated from an I. ricinus tick in Switzerland, has multi-locus sequence type (MLST) 679, strain ID number 1887 in the Borrelia MLST database, and ospC major group (oMG) A10. Strain Fin-Jyv-A3 was isolated from a Finnish bank vole, has MSLT 676, strain ID number 1961 in the Borrelia MLST database and oMG A3. We determined that both strains contain all the plasmids necessary to complete the life cycle of $B$. afzelii (see section 1 in the electronic supplementary material (ESM)).

Our previous work has shown that $B$. afzelii can invade a diversity of tissues including the bladder, heart, ear, joints, and skin, and that the spirochete loads in internal organs (e.g., bladder and heart) are generally lower than external organs (ear and skin) [33, 36, 37]. In our previous work with strains Fin-Jyv-A3 and NE4049, we did not directly compare the tissue infection prevalence or tissue spirochete load between these two strains [36, 37], and we therefore have no a priori predictions with respect to these two phenotypes.

\section{Ethics approval}

The commission that is part of the Service de la Consommation et des Affaires Vétérinaires (SCAV) of Canton Vaud, Switzerland evaluated and approved the ethics of this study. The veterinary service of the Canton of Neuchâtel, Switzerland issued the animal experimentation permit used in this study (NE04/2016).

\section{Experimental infection of mice via tick bite with one or two strains of $B$. afzelii}

The study consisted of two independent experiments that differed with respect to the focal strain. In experiment 1 , the focal strain was Fin-Jyv-A3; 10 mice were infected with strain Fin-Jyv-A3 (single infection) and 10 mice were simultaneously co-infected with strains Fin-Jyv-A3 and 
NE4049 (coinfection). In experiment 2, the focal strain was NE4049; 10 mice were infected with strain NE4049 (single infection) and 10 mice were simultaneously co-infected with strains NE4049 and Fin-Jyv-A3 (coinfection). Thus, across the two experiments, 10 mice were infected with strain Fin-Jyv-A3, 10 mice were infected with strain NE4049, and 20 mice were co-infected with both strains. All mice were infected via tick bite by using I. ricinus nymphs that had been experimentally infected with one of the two B. afzelii strains of interest (see section 2 in the ESM). Mice in the coinfection treatment were simultaneously infested with five nymphs infected with strain FinJyv-A3 and five nymphs infected with strain NE4049. Mice in the single infection treatment were infested with five nymphs, infected with the focal strain and five uninfected nymphs, so that each mouse was infested with a total of 10 nymphs. To confirm $B$. afzelii infection, we took a blood sample at 4 weeks post-infection (PI) and used the SERION ELISA classic Borrelia burgdorferi IgG/IgM immunoassay to detect Borrelia-specific IgG antibodies.

\section{Host-to-tick transmission of B. afzelii}

To measure host-to-tick transmission, mice were infested with I. ricinus larvae at 5 weeks PI as previously described $(14,15)$. The engorged larvae were allowed to moult into nymphs, which were sacrificed by freezing. For each mouse, a sample of 20 nymphs (range $=7-25$ ) was tested for the two strains of B. afzelii as previously described (14, 15). For each mouse, the percentage of nymphs infected with the focal strain (Fin-Jyv-A3 or NE4049) is an estimate of the strain-specific transmission success.

\section{Mice dissection and DNA extraction}

At 6 weeks PI, mice were euthanized with $\mathrm{CO}_{2}$. Mice were dissected, and six mouse organs were collected: bladder, left ear, right ear, heart, right ankle joint, and the section of dorsal skin where the nymphs had attached. For the left ear, right ear and dorsal skin, $\sim 1 \mathrm{mg}$ of tissue was sampled using a type II forceps ( $2 \mathrm{~mm}$ diameter). For the bladder, heart, and ankle joint, we used a scalpel to cut $\sim 20 \mathrm{mg}$ of tissue. DNA from the mouse tissue samples was extracted with Qiagen DNeasy Blood and Tissue kit 96-well plates and following the Qiagen protocol. Extracted DNA was eluted in $150 \mu \mathrm{l}$ of autoclaved milliQ water.

\section{Flagellin qPCR}

The total spirochete load in the mouse tissues was estimated using a qPCR that targeted a 132-bp fragment of the flagellin gene (33) using a previously described protocol (14) (see section 2 in the ESM). All samples were processed three times, each of the three replicates was performed in a different plate on a different day. All the plates contained three negative DNA extraction controls (tissues from uninfected mice), two negative controls for the qPCR (PCRgrade water), and four standards containing $10^{2}, 10^{3}, 10^{4}$, and $10^{5}$ copies of the flagellin gene. For the three independent qPCR assays, the overall repeatability of the $\mathrm{Cq}$ values was $86.9 \%$. The mean of the $\mathrm{Cq}$ values of the three independent qPCRs was used to calculate the mean total spirochete load in $3 \mu \mathrm{l}$ of DNA template for each of the mouse tissue samples.

\section{Nested strain-specific qPCRs}

The two strains used in this study can be differentiated using the $o s p C$ gene; strains Fin-Jyv-A3 and NE4049 carry ospC major group alleles A3 and A10, respectively. The strainspecific spirochete load was estimated using two independent qPCR assays that targeted a 142-bp fragment of the $\operatorname{sspC}$ gene. However, because the spirochete load was very low in the mouse tissues, we first used a conventional PCR to enrich the number of $o s p C$ gene copies relative to the amount of mouse DNA. This PCR amplified a 657-bp fragment of the $o s p C$ gene using a previously described protocol [24] (see section 2 in the ESM). The amplicons from the conventional PCR were the template for the strain-specific $\operatorname{ssp} C \mathrm{qPCR}$, and for this reason, the results from the latter were used to estimate the relative abundance (and not the absolute abundance) of the two strains in the tissue samples.

The amplicons from the conventional PCR were run in triplicate in the $o s p C \mathrm{~A} 3 \mathrm{qPCR}$ and in the $o s p C \mathrm{~A} 10 \mathrm{qPCR}$ to determine which strains were present. The primers that amplify the 142-bp fragment of the $o s p C$ gene are the same for the two strains; strain specificity is determined by the probes, which are different for each $o s p C$ allele. This strainspecific qPCR protocol has been described elsewhere [14] (see section 2 in the ESM).

\section{Repeatability of the strain-specific spirochete load}

The repeatability of the strain-specific spirochete load was estimated by performing all three qPCR reactions in triplicate on the same set of 222 organ tissue samples. The overall repeatability (across all 222 organ tissue samples) of the spirochete load of strain Fin-Jyv-A3 and strain NE4049 was $78.9 \%$ and $87.0 \%$, respectively (see section 2 in the ESM for details).

\section{Statistical Analysis}

The statistical analyses were done using $\mathrm{R}$ version 1.2.5019 and the following packages: base, car, lme4 and emmeans. 
The lmer() and glmer() functions (lme4 package) were used to create the generalized linear mixed effects models (GLMMs) and linear mixed effects models (LMMs). The Anova() function (car package) and the anova() function (base package) were used to perform the log-likelihood ratio tests. The emmeans() and the pairs() functions (emmeans package) were used for the post-hoc analysis of the GLMMs and LMMs.

\section{Analysis of the $B$. afzelii tissue infection prevalence}

The flagellin qPCR (which cannot distinguish between strains) was used to determine whether tissues were infected with B. afzelii or not. Tissue samples were considered infected with $B$. afzelii if at least two of the three replicate flagellin qPCR assays tested positive. The binomial response variable was whether $B$. afzelii was absent (0) or present (1) in a tissue sample, and it was analysed as a GLMM with binomial errors.

\section{Analysis of the total tissue spirochete load}

The flagellin qPCR (which cannot distinguish between strains) was used to estimate the total B. afzelii spirochete load per $\mathrm{mg}$ of tissue in each mouse organ (or per mg of DNA). The spirochete loads estimated by the flagellin qPCR in $3 \mu \mathrm{l}$ of DNA template were corrected to the total DNA extraction elution volume $(150 \mu \mathrm{l})$, standardized by mg of tissue extracted (or by the DNA concentration of the DNA extraction), and $\log 10$ transformed to improve normality. The spirochete loads standardized by $\mathrm{mg}$ of tissue were highly correlated with the spirochete loads standardized by mg of DNA ( $r=0.946, p<0.001$; see section 3 in the ESM), and for brevity, only the former are shown in the main manuscript. The standardized $\log 10$-transformed tissue spirochete load was analysed as an LMM.

\section{Effect of strain, coinfection, and mouse organ on the strain-specific tissue infection prevalence}

The focal strains in experiments 1 and 2 are Fin-Jyv-A 3 and NE4049, respectively. In experiments 1 and 2, the tissue sample was considered infected with the focal strain (FinJyv-A3 or NE4049) if at least two of the three replicate strain-specific qPCR assays (ospC type A3 or ospC type A10) tested positive. Thus, in experiments 1 and 2, the response variable is whether the mouse tissues tested positive for $o s p C$ type A3 versus $o s p C$ type A10, respectively, as determined by the two different strain-specific qPCR assays. The binomial response variable was whether the focal strain was absent (0) or present (1) in a tissue sample, and it was analysed as a GLMM with binomial errors. The fixed factors were focal strain (two levels:
Fin-Jyv-A3, NE4049), coinfection (two levels: no, yes), mouse organ (six levels: bladder, left ear, right ear, heart, joint, and dorsal skin), and their interactions. Mouse identity was modeled as a random factor. Models that differed with respect to the fixed factor of interest were compared using log-likelihood ratio (LLR) tests to determine statistical significance.

\section{Effect of strain, coinfection, and mouse organ on the strain-specific tissue spirochete load}

The focal strains in experiments 1 and 2 are Fin-Jyv-A3 and NE4049, respectively. For the co-infected mice, the abundance of each strain in a given tissue was estimated by combining the total spirochete abundance of the flagellin qPCR with the relative abundances of the ospC qPCRs (see section 2 in the ESM for details). Thus, in experiments 1 and 2 , the response variable is the spirochete load of strain Fin-Jyv-A3 versus strain NE4049, respectively. These strain-specific spirochete loads were $\log 10$ transformed to improve normality and this variable was analysed using an LMM. The fixed factors, random factors, model simplification, and model analysis were the same as the analysis for the strain-specific tissue infection prevalence.

\section{Results}

Eight mice were excluded from the study: two mice died during the experiment, one mouse was not infected, and five mice in the co-infected group only became infected with strain Fin-Jyv-A3. We believe that these five mice were not properly exposed to strain NE4049 (e.g., nymphs escaped from the capsule or refused to feed on the mice). If we include these five mice, our results become more significant because strain NE4049 is 'excluded' by strain Fin-Jyv-A3. The conservative approach is therefore to exclude these five mice. Thus, the final sample size consisted of 32 mice: strain Fin-Jyv-A3 alone $(n=9)$, strain Fin-Jyv-A3 in coinfection $(n=7)$, strain NE4049 alone $(n=9)$, and strain NE4049 in coinfection $(n=7)$.

\section{The $B$. afzelii infection prevalence differs among mouse organs}

We estimated the prevalence of $B$. afzelii infection in the tissue samples of the six mouse organs $(n=192$ tissue samples for 32 mice) using the flagellin qPCR. The rank order of tissue infection prevalence among the six mouse organs (from highest to lowest) was bladder $(32 / 32=$ $100.0 \%)$, left ear $(29 / 32=90.6 \%)$, right ear $(29 / 32=$ $90.6 \%)$, heart $(27 / 32=84.4 \%)$, ankle joint $(28 / 32=87.5 \%)$, and dorsal skin $(20 / 32=62.5 \%)$. The significant differences 
in tissue infection prevalence among organs $(p<0.001)$ indicate that $B$. afzelii exhibits tissue tropism (see section 4 in the ESM for a comprehensive analysis).

\section{The B. afzelii infection prevalence differs among strains}

We tested whether the two B. afzelii strains differed in tissue tropism for the subset of mice infected with one strain $(n=18)$. We used a GLMM with binomial errors to analyse the tissue infection prevalence as a function of mouse organ and strain. The interaction between focal strain and organ was not significant $(p=0.286)$. The tissue infection prevalence for strain NE4049 $(90.7 \%=49 / 54)$ was significantly higher $(1.2 \mathrm{x})$ than that of strain Fin-Jyv-A3 $(74.1 \%=40 / 54 ; p=0.015)$. Thus, strain NE4049 was more successful at establishing infection in rodent tissues than strain Fin-Jyv-A3. As shown previously, the tissue infection prevalence differed significantly among mouse organs $(p=$ $0.024)$.

\section{The total tissue spirochete load of $B$. afzelii differs among mouse organs}

We estimated the spirochete load of $B$. afzelii in the subset of infected mouse tissue samples $(n=165$ tissue samples for 32 mice; uninfected tissues were excluded) using the flagellin qPCR. The heart, which had the lowest spirochete load, was set as the reference; the spirochete load was higher in the bladder (5.2x), ankle joint $(5.4 \mathrm{x})$, dorsal skin $(25.7 x)$, right ear $(122.2 x)$, and left ear $(147.7 x)$, and these differences among mouse organs were highly significant (Fig. 1; $p<0.000001$; see section 5 in the ESM for the complete analysis). The results were similar when the spirochete loads were standardized per mg of DNA (see section 5 in the ESM). The matrix of pairwise correlations in the total spirochete load between the six mouse organs found that the highest correlations were for pairs of skinrelated organs (see section 6 in the ESM).

\section{The total tissue spirochete load differs among strains}

We tested whether the two B. afzelii strains differed in tissue bacterial abundance for the subset of mice infected with one strain $(n=89$ tissue samples for $n=18$ mice; uninfected tissues were excluded). We used an LMM to analyse the standardized $\log 10$-transformed strain-specific tissue spirochete loads as a function of mouse organ and focal strain. The interaction between focal strain and mouse organ was significant $(p=0.037)$, and we therefore compared the tissue spirochete load between the two strains separately for each mouse organ. The mean

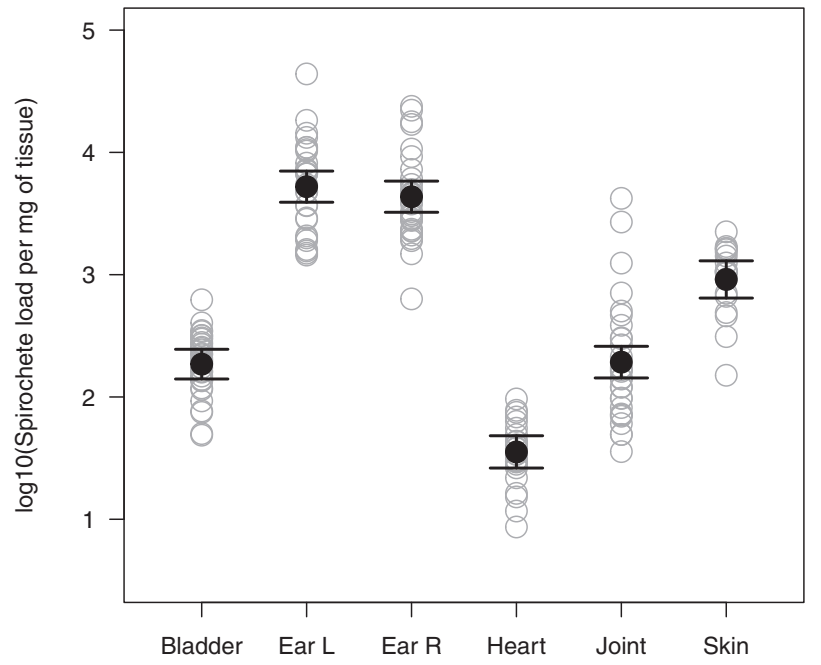

Fig. 1 The spirochete load of $\boldsymbol{B}$. afzelii differs among the six mouse organs. The tissue spirochete load was estimated using a flagellin qPCR and was standardized per mg of tissue. The log10-transformed spirochete load per mg of tissue is significantly different among the six mouse organs: bladder, left ear (Ear L), right ear (Ear R), heart, right ankle joint, and the section of dorsal skin where the nymphs had attached. Shown are the means, the $95 \%$ confidence intervals, and the individual data points.

spirochete load in the ankle joints for strain Fin-Jyv-A3 (mean $=347 ; 95 \% \mathrm{CI}=184-655$; units $=$ spirochetes per $\mathrm{mg}$ of tissue) was significantly higher (3.8x) than that of strain NE4049 $($ mean $=98 ; 95 \% \quad \mathrm{CI}=61-158 ; \quad p=$ 0.0016). There were no significant differences in spirochete load between the two strains for the other five mouse organs (see section 7 in the ESM). Thus, strain FinJyv-A3 established higher spirochete loads in the joint tissues than strain NE4049.

\section{Effect of strain, coinfection, and mouse organ on the strain-specific tissue infection prevalence}

The strain-specific tissue infection prevalence refers to whether a tissue was infected with the focal strain. We used a GLMM with binomial errors to analyse the strain-specific infection prevalence in the mouse tissues as a function of focal strain, coinfection and mouse organ (see section 8 in the ESM for the complete analysis). Non-significant terms were removed using stepwise model simplification. The interaction between strain and coinfection was almost significant (Fig. 2; $p=0.080$ ) and we therefore split the analysis by strain. Coinfection in the rodent host significantly reduced the number of mouse organs infected with strain Fin-Jyv-A3 (Fig. 2; $p=0.00007$ ). In contrast, coinfection in the rodent host had no effect on the number of mouse organs infected with strain NE4049 (Fig. 2; $p=$ 0.446). Thus, coinfection resulted in competition between strains, which reduced the presence of strain Fin-Jyv-A3, 

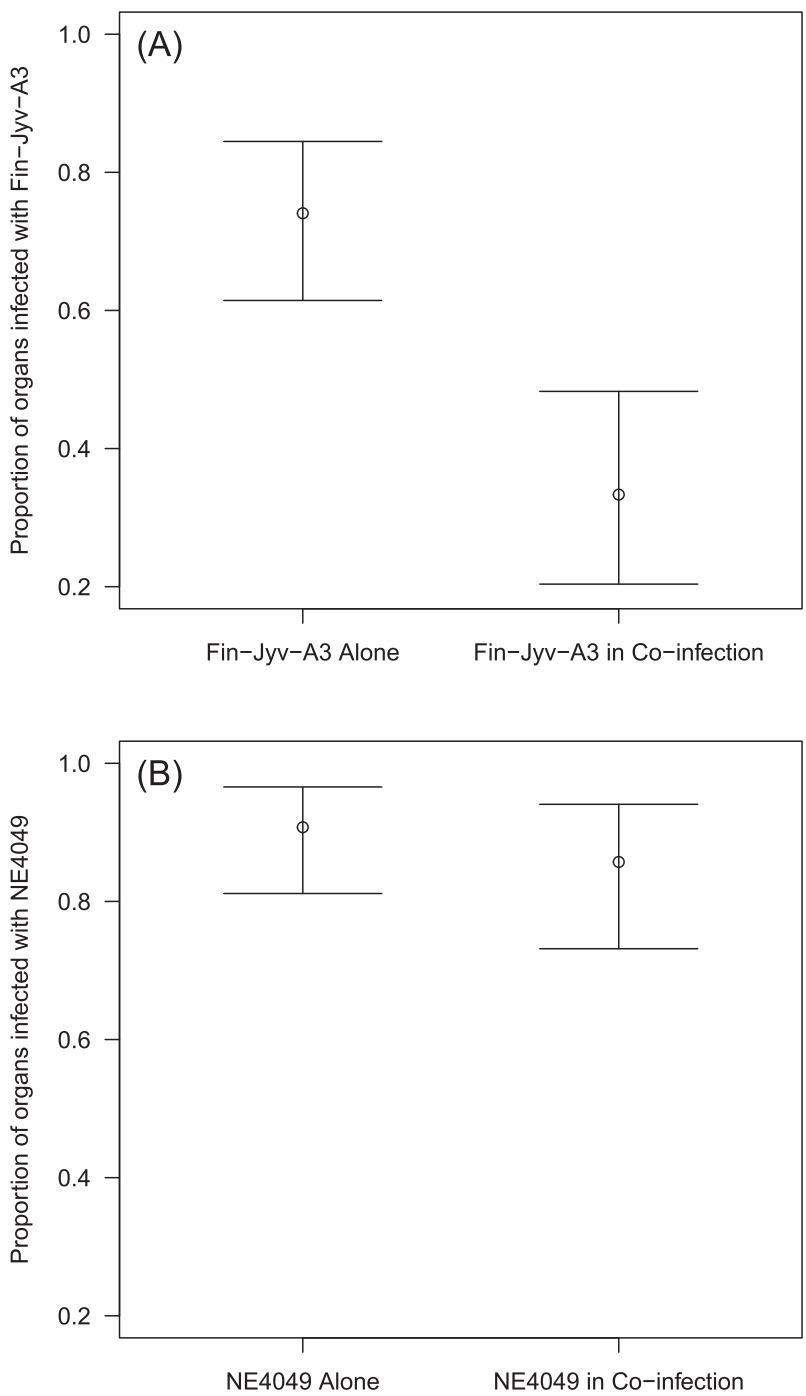

Fig. 2 Coinfection in the rodent host influences the proportion of mouse organs infected by the focal strain. Panels $\mathbf{A}$ and $\mathbf{B}$ show the results from two independent experiments where the focal strains are Fin-Jyv-A3 and NE4049, respectively. The interaction between focal strain and coinfection was almost significant $(p=0.080)$. When the strains were analysed separately, coinfection reduced the strainspecific prevalence significantly for $(\mathbf{A})$ strain Fin-Jyv-A3 $(p<0.0001)$ but not for (B) strain NE4049 $(p=0.445)$. Shown are the means and the $95 \%$ confidence intervals.

but not strain NE4049, in the host tissues (see section 9 in the ESM for an additional analysis).

\section{Effect of strain, coinfection, and mouse organ on the strain-specific tissue spirochete load}

For the subset of infected mouse tissue samples $(n=165)$, we used an LMM to analyse the strain-specific spirochete load in the mouse tissue samples as a function of focal strain, coinfection and mouse organ (see section 10 in the ESM for the complete analysis). A classic stepwise model simplification approach found that the three-way interaction was significant $(p=0.031)$, and the analysis was therefore divided by focal strain. For strain Fin-Jyv-A3, the interaction between coinfection and mouse organ was significant $(p=0.0002)$; we therefore tested the effect of coinfection separately for each mouse organ. In the bladder, coinfection significantly decreased (4.0x) the mean spirochete load of strain Fin-Jyv-A3 $(p=0.003)$. In the ankle joint, coinfection significantly increased $(5.9 \mathrm{x})$ the mean spirochete load of strain Fin-Jyv-A3 $(p=0.022)$. The contrast between single infection and coinfection was not significant for the other three mouse organs. For strain NE4049, the interaction between coinfection and mouse organ was not significant $(p=0.677)$. Coinfection was not significant $(p=$ 0.061 ), but as before, mouse organ had a significant effect on the strain-specific tissue spirochete load $(p<0.000001)$. The results were similar when the tissue spirochete loads were standardized per $\mathrm{mg}$ of DNA (see section 11 in the ESM).

\section{Effects of coinfection and strain on host-to-tick transmission of the focal strain}

We used a GLMM with binomial errors to test whether strain and coinfection influenced the strain-specific host-totick transmission success. The interaction between strain and coinfection was significant (Fig. 3; $p=0.028$ ), and we therefore split the analysis by strain. For strain Fin-Jyv-A3, coinfection significantly reduced host-to-tick transmission (Fig. 3; $p=0.0006$ ). In contrast, for strain NE4049, coinfection had no effect on host-to-tick transmission (Fig. 3; $p=0.492$ ).

\section{Relationship between host-to-tick transmission of the focal strain and the presence and abundance of the focal strain in the mouse organs}

We used a GLMM with binomial errors to test whether variation in the strain-specific host-to-tick transmission success depended on either (1) the number of mouse organs infected with the focal strain, or (2) the mean spirochete load of the focal strain for the subset of infected mouse organs.

The positive relationship between the number of mouse organs infected with the focal strain and host-to-tick transmission of the focal strain was highly significant (Fig. 4(A); $p=0.0005$; see section 12 in the ESM). Similarly, the positive relationship between the mean spirochete load of the focal strain for the subset of infected mouse organs and host-to-tick transmission of the focal strain was highly significant (Fig. 4(B); $p=0.003$; see section 13 in the ESM). After correcting for differences among organs, the positive relationship between the mean 

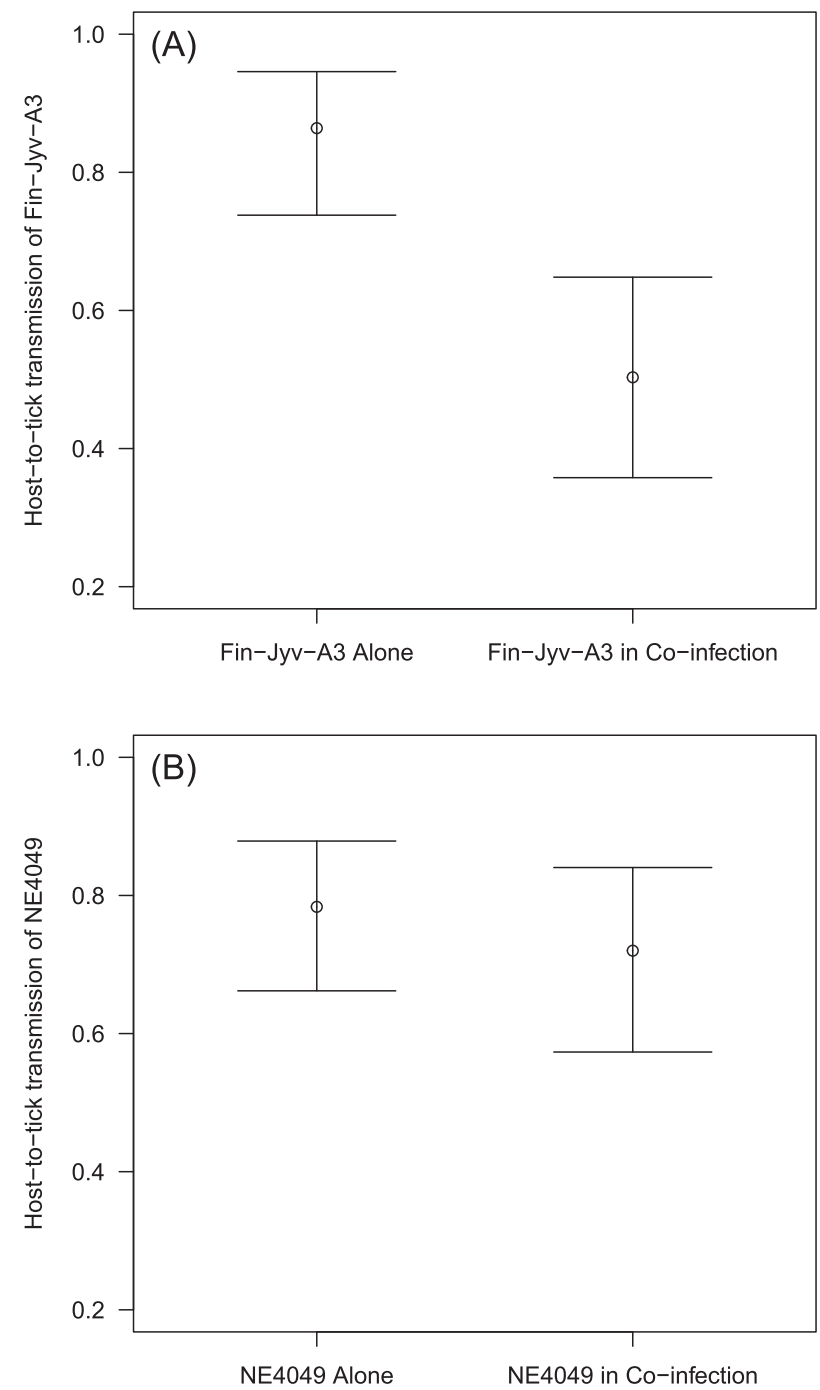

Fig. 3 Coinfection in the rodent host influences the host-to-tick transmission of the focal strain. Panels $\mathbf{A}$ and $\mathbf{B}$ show the results from two independent experiments where the focal strains are Fin-JyvA3 and NE4049, respectively. The interaction between focal strain and coinfection was significant $(p=0.028)$, and we therefore split the analysis by strain. Coinfection reduced host-to tick transmission for both strains; the effect was significant for (A) strain Fin-Jyv-A3 ( $p=$ $0.0006)$, but not for $(\mathbf{B})$ strain NE4049 $(p=0.492)$. Shown are the means and the $95 \%$ confidence intervals.

spirochete load of the focal strain for the subset of infected organs and host-to-tick transmission of the focal strain was almost significant ( $p=0.050$; see section 13 in the ESM).

\section{Discussion}

\section{Prevalence of $B$. afzelii in the tissues of the rodent host}

Overall, the prevalence of $B$. afzelii infection in the mouse organs was high; five of the six mouse organs had an
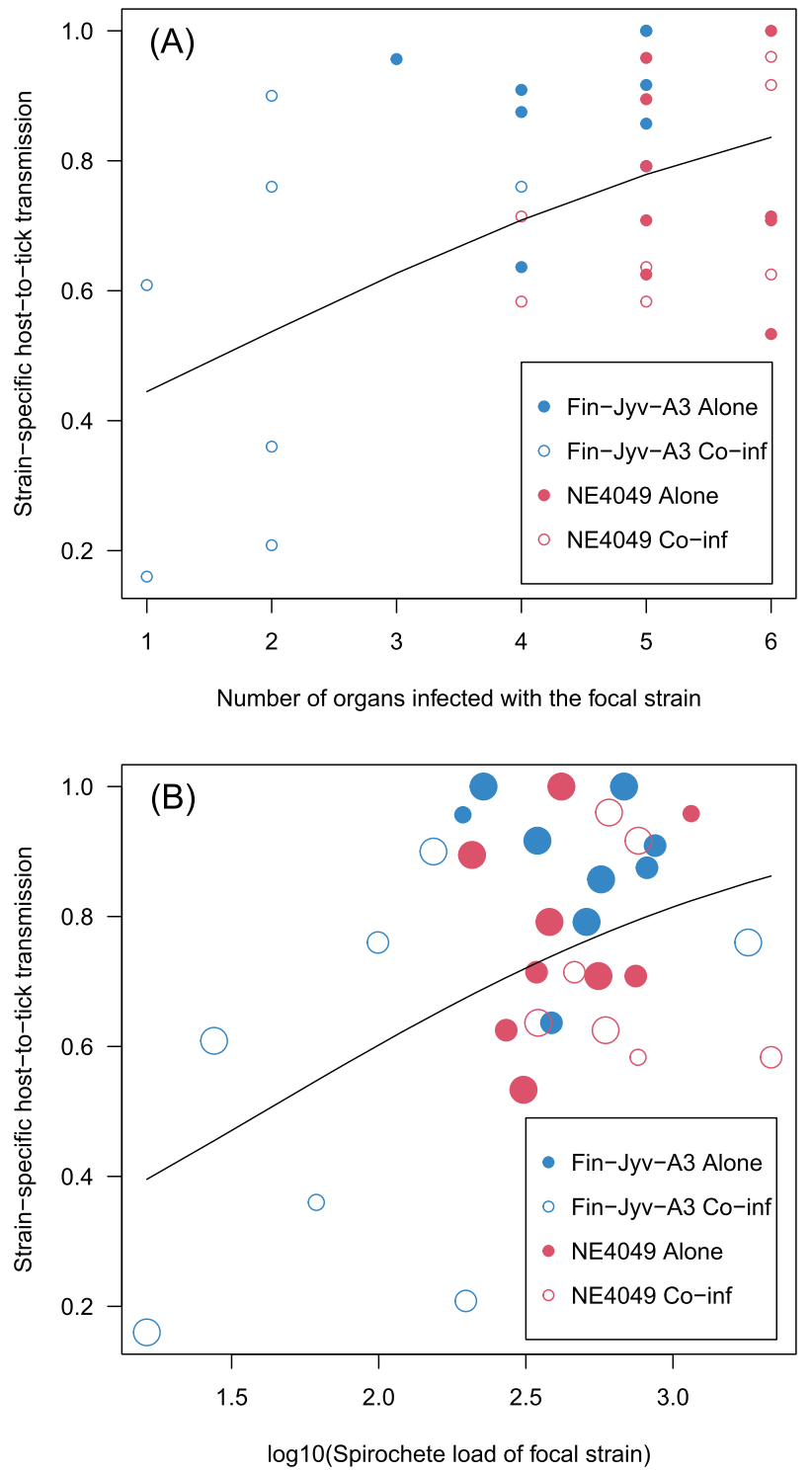

Fig. 4 Host-to-tick transmission of the focal strain depends on the number of mouse organs that are infected with the focal strain or the mean spirochete load of the focal strain in the mouse organs. (A) The six mouse organs included the bladder, left ear, right ear, heart, right ankle joint, and the section of dorsal skin where the nymphs had attached. (B) The log10-transformed spirochete load of the focal strain was averaged over the subset of infected organs (the size of each circle indicates the number of infected organs). Strain Fin-Jyv-A3 and strain NE4049 are shown in blue and red colours, respectively. Mice in the single strain groups and the co-infected groups are shown with solid and empty symbols, respectively. The black line shows the line of best fit from a generalized linear model (GLM) with binomial errors.

infection prevalence $>80.0 \%$. These results were similar to a recent experimental infection study with strain NE4049 in the same host (27 female BALB/c mice) where $92.6 \%$ of the tissue samples (100/108) tested positive for B. afzelii (34). In the present study, we found that NE4049 established infection in more tissues than Fin-Jyv-A3, which suggests that NE4049 is more invasive or more infectious than Fin-Jyv-A3. 


\section{Spirochete load of $B$. afzelii in the tissues of the rodent host}

The spirochete load of $B$. afzelii per mg of tissue differed greatly between the mouse organs. For the subset of infected tissues, we found that the spirochete load was highest in the ears and the dorsal skin, and lower in internal organs like the heart, bladder, and ankle joints. Other studies on B. afzelii and B. burgdorferi sensu stricto (ss) in rodent hosts, have also found that the spirochete load is higher in the skin compared to internal organs like the bladder and heart [33, 36-39]. One explanation for this result is that the skin is important for the host-to-tick transmission of $B$. burgdorferi sl [21-23], whereas the internal organs (e.g., bladder, heart, and joints) are a deadend for spirochete transmission to ticks (39).

For the mice that were infected with single strains, we found that strain Fin-Jyv-A3 established a higher spirochete load in the ankle joints compared to strain NE4049. Previous studies on B. afzelii and B. burgdorferi ss in lab mice have shown differences in tissue spirochete load between strains $[21,38,40,41]$. This strain-specific variation is important from a medical perspective because it has been associated with host pathology including carditis, arthritis, and ankle joint swelling [40, 41]. The higher abundance of Fin-Jyv-A3 in the joints suggests that this strain would cause more arthritis and joint swelling in the host compared to NE4049, as observed for strains of B. burgdorferi ss in lab mice $[40,41]$.

\section{Competition between strains of $B$. afzelii in the tissues of the rodent host}

Our study demonstrated that coinfection of mice with two strains of $B$. afzelii resulted in competitive interactions between strains in the host tissues. We measured the outcome of coinfection in two ways: (1) strain-specific tissue infection prevalence and (2) strain-specific tissue spirochete load. For strain Fin-Jyv-A3, coinfection reduced the tissue infection prevalence by $55 \%$, indicating a strong effect of competition. The results of our study agree with other studies on vector-borne pathogens that have shown that coinfection leads to competitive interactions in the vertebrate host [3, 10, 42]. Our study is the first experimental demonstration that strains belonging to the same B. burgdorferi sl genospecies compete inside the tissues of the vertebrate host. An uncontrolled field study in Sweden on small mammals naturally infected with $B$. afzelii previously found results consistent with inter-strain competition in the host [11]. Other studies on B. afzelii and B. burgdorferi ss have shown that coinfection in the rodent host reduced strain-specific transmission to ticks [14, 15, 32, 43], however, this result could be caused by interactions in the rodent host, the tick vector, or both. In contrast, our controlled study clearly shows that coinfection and competitive interactions reduce the ability of some $B$. afzelii strains to establish infection in the tissues of the rodent host.

With respect to tissue spirochete load, we found that the outcome of the interactions between strains depended on the mouse organ. For strain Fin-Jyv-A3, coinfection in the mouse host had opposite effects on the bacterial abundance in the bladder (decrease) and ankle joints (increase). We had expected that inter-strain competition would be most intense in the ears and dorsal skin because these organs are critical for host-to-tick transmission, but this was not the case. Our study shows the importance of sampling numerous organs when testing whether coinfection influences the strainspecific abundance in the tissues of the vertebrate host.

Our study does not elucidate the mechanism of competition, which includes interference, exploitation, and apparent competition [6, 7, 44]. One plausible mechanism is exploitation competition, which occurs when pathogen strains compete over limited host resources such as nutrients or space $[42,45,46]$. Another plausible mechanism is apparent competition, where the host immune system mediates the interactions between strains [47, 48]. Interference competition is unlikely because this mechanism requires the production of toxic substances that weaken the performance of other strains, but B. burgdorferi sl does not produce such toxins [49].

\section{Pathogen distribution in host tissues and host-to- vector transmission}

Host-to-vector transmission is one of the critical life history traits for any vector-borne pathogen and determines the frequency of a strain in nature and whether it is common or rare $[28,35,50]$. As we have shown previously [14, 15], coinfection in the rodent host and competition between strains reduced the host-to-tick transmission of the focal strain. What is novel about this study is our demonstration that inter-strain competition reduced the presence of the two strains in the rodent tissues, which in turn, reduced their host-to-tick transmission success. Further evidence for the link between tissue pathogen loads and transmission was our demonstration that both the mean tissue infection prevalence and the mean tissue spirochete load were positively related to host-to-tick transmission of the focal strain. Our study agrees with studies on the rodent malaria parasite ( $P$. chabaudi) in lab mice, where strains with higher abundance in the blood have higher mouse-to-mosquito transmission [3, 18, 51, 52]. Previous studies on B. afzelii found a positive relationship between the spirochete load in rodent ear tissues and host-to-tick transmission [21, 22], but these studies did not investigate inter-strain competition. Our study is the first experimental demonstration that 
competitive interactions between strains of B. burgdorferi sl in the host tissues determine strain-specific host-to-tick transmission success.

\section{Inter-strain competition and strain diversity of $B$. burgdorferi sl pathogens in nature}

In nature, there is substantial strain diversity of $B$. burgdorferi sl pathogens at small spatial scales [26, 29, 53, 54]. Studies have shown that some strains are much more common than others, and that these differences in strain frequency are constant over time and space $[28,30,55,56]$. A major question is to understand the ecological factors that maintain the strain composition of the pathogen population. The multiple niche polymorphism (MNP) hypothesis proposes that the different strains are adapted to different vertebrate reservoir hosts $[53,57]$. The present study suggests that competitive interactions between strains in the host tissues can also have important consequences for their host-to-tick transmission. The MNP hypothesis and the inter-strain competition hypothesis are not mutually exclusive; for example, the competitive ability of strains could differ between vertebrate hosts. Future studies should investigate the relative importance of MNP versus interstrain competition in maintaining the strain diversity of $B$. burgdorferi sl pathogens in nature.

\section{Conclusions}

For multi-strain vector-borne pathogens, we show that competition between strains in the host tissues can reduce host-to-vector transmission. Simultaneous coinfection of the rodent host can inhibit the ability of strains to invade and establish infection in the host tissues. Depending on the organ, coinfection and interactions between strains either increased or decreased the spirochete abundance of a given strain. The ability to establish infection in many tissues and the mean bacterial abundance were both positively related with strain-specific host-to-tick transmission success.

Acknowledgements The authors sincerely thank Kheirie Kabalan and Alessandro Belli for their help during the study. This work was supported by the following grants awarded to Maarten J. Voordouw: a Swiss National Science Foundation grant (FN 31003A_141153), a Discovery Grant from the Natural Sciences and Engineering Research Council of Canada (RGPIN-2019-04483), and an Establishment Grant from the Saskatchewan Health Research Foundation (4583). This study is part of the $\mathrm{PhD}$ thesis of Dolores Genné.

Author contributions DG and MJV conceived and designed the study. DG conducted the experiment. DG, MR, AS, and FB performed the molecular work. MR and AS developed the nested strain-specific qPCRs. OR helped with the experimental infections of the mice. ROMR performed the plasmid profile analysis of the B. afzelii strains. DG performed the statistical analysis. DG and MJV wrote the manuscript. All authors read and approved the final version of the manuscript.

Funding Open Access funding provided by Université de Neuchâtel.

\section{Compliance with ethical standards}

Conflict of interest The authors declare no competing interests.

Publisher's note Springer Nature remains neutral with regard to jurisdictional claims in published maps and institutional affiliations.

Open Access This article is licensed under a Creative Commons Attribution 4.0 International License, which permits use, sharing, adaptation, distribution and reproduction in any medium or format, as long as you give appropriate credit to the original author(s) and the source, provide a link to the Creative Commons license, and indicate if changes were made. The images or other third party material in this article are included in the article's Creative Commons license, unless indicated otherwise in a credit line to the material. If material is not included in the article's Creative Commons license and your intended use is not permitted by statutory regulation or exceeds the permitted use, you will need to obtain permission directly from the copyright holder. To view a copy of this license, visit http://creativecommons. org/licenses/by/4.0/.

\section{References}

1. Read AF, Taylor LH. The ecology of genetically diverse infections. Science. 2001;292:1099-102.

2. Balmer O, Tanner M. Prevalence and implications of multiplestrain infections. Lancet Infect Dis. 2011;11:868-78.

3. de Roode JC, Pansini R, Cheesman SJ, Helinski MEH, Huijben S, Wargo AR, et al. Virulence and competitive ability in genetically diverse malaria infections. Proc Natl Acad Sci USA. 2005;102: 7624-8.

4. de Roode JC, Yates AJ, Altizer S. Virulence-transmission tradeoffs and population divergence in virulence in a naturally occuring butterfly parasite. Proc Natl Acad Sci USA. 2008;105:7489-94.

5. Alizon S, de Roode JC, Michalakis Y. Multiple infections and the evolution of virulence. Ecol Lett. 2013;16:556-67.

6. Mideo N. Parasite adaptations to within-host competition. Trends Parasitol. 2009;25:261-8.

7. Bashey F. Within-host competitive interactions as a mechanism for the maintenance of parasite diversity. Philos T R Soc B. 2015;370:1-8.

8. Alizon S, Lion S. Within-host parasite cooperation and the evolution of virulence. P R Soc B-Biol Sci. 2011;278:3738-47.

9. Andersson M, Scherman K, Raberg L. Multiple-strain infections of Borrelia afzelii: a role for within-host interactions in the maintenance of antigenic diversity? Am Nat. 2013;181:545-54.

10. Balmer O, Stearns SC, Schotzau A, Brun R. Intraspecific competition between co-infecting parasite strains enhances host survival in African trypanosomes. Ecology. 2009;90:3367-78.

11. Strandh M, Raberg L. Within-host competition between Borrelia afzelii ospC strains in wild hosts as revealed by massively parallel amplicon sequencing. Philos T Roy Soc B. 2015;370:1-8.

12. Bell AS, De Roode JC, Sim D, Read AF. Within-host competition in genetically diverse malaria infections: parasite virulence and competitive success. Evolution. 2006;60:1358-71.

13. de Roode JC, Culleton R, Cheesman SJ, Carter R, Read AF. Host heterogeneity is a determinant of competitive exclusion or 
coexistence in genetically diverse malaria infections. P R Soc BBiol Sci. 2004;271:1073-80.

14. Genné D, Sarr A, Gomez-Chamorro A, Durand J, Cayol C, Rais O et al. Competition between strains of Borrelia afzelii inside the rodent host and the tick vector. P Roy Soc B-Biol Sci. 2018;285: $1-10$.

15. Genné D, Sarr A, Rais O, Voordouw MJ. Competition between strains of Borrelia afzelii in immature Ixodes ricinus ticks is not affected by season. Front Cell Infect Microbiol. 2019;9:1-14.

16. Pollitt LC, Bram JT, Blanford S, Jones MJ, Read AF. Existing infection facilitates establishment and density of malaria parasites in their mosquito vector. PLOS Pathog. 2015;11:1-18.

17. Reif KE, Palmer GH, Crowder DW, Ueti MW, Noh SM. Restriction of Francisella novicida genetic diversity during infection of the vector midgut. PLOS Pathog. 2014;10:1-11.

18. Schneider P, Bell AS, Sim DG, O'Donnell AJ, Blanford S, Paaijmans KP, et al. Virulence, drug sensitivity and transmission success in the rodent malaria, Plasmodium chabaudi. P R Soc BBiol Sci. 2012;279:4677-85.

19. van Duijvendijk G, Sprong H, Takken W. Multi-trophic interactions driving the transmission cycle of Borrelia afzelii between Ixodes ricinus and rodents: a review. Parasit Vectors. 2015;8:1-11.

20. Rollend L, Fish D, Childs JE. Transovarial transmission of Borrelia spirochetes by Ixodes scapularis: A summary of the literature and recent observations. Ticks Tick Borne Dis. 2013;4:46-51.

21. Jacquet M, Durand J, Rais O, Voordouw MJ. Cross-reactive acquired immunity influences transmission success of the Lyme disease pathogen, Borrelia afzelii. Infect Genet Evolution. 2015;36:131-40.

22. Raberg L. Infection intensity and infectivity of the tick-borne pathogen Borrelia afzelii. J Evol Biol. 2012;25:1448-53.

23. Grillon A, Westermann B, Cantero P, Jaulhac B, Voordouw MJ, Kapps D, et al. Identification of Borrelia protein candidates in mouse skin for potential diagnosis of disseminated Lyme borreliosis. Sci Rep. 2017;7:1-13.

24. Bunikis J, Garpmo U, Tsao J, Berglund J, Fish D, Barbour AG. Sequence typing reveals extensive strain diversity of the Lyme borreliosis agents Borrelia burgdorferi in North America and Borrelia afzelii in Europe. Microbiol-Sgm. 2004;150:1741-55.

25. Lagal V, Postic D, Ruzic-Sabljic E, Baranton G. Genetic diversity among Borrelia strains determined by single-strand conformation polymorphism analysis of the $\operatorname{sp} C$ gene and its association with invasiveness. J Clin Microbiol. 2003;41:5059-65.

26. Durand J, Jacquet M, Paillard L, Rais O, Gern L, Voordouw MJ. Cross-immunity and community structure of a multiple-strain pathogen in the tick vector. Appl Environ Microbiol. 2015;81:7740-52.

27. Durand J, Herrmann C, Genné D, Sarr A, Gern L, Voordouw MJ. Multistrain infections with Lyme borreliosis pathogens in the tick vector. Appl Environ Microbiol. 2017;83:1-14.

28. Durand J, Jacquet M, Rais O, Gern L, Voordouw MJ. Fitness estimates from experimental infections predict the long-term strain structure of a vector-borne pathogen in the field. Sci Rep. 2017;7: $1-9$.

29. Hellgren O, Andersson M, Raberg L. The genetic structure of Borrelia afzelii varies with geographic but not ecological sampling scale. J Evol Biol. 2011;24:159-67.

30. Raberg L, Hagstrom A, Andersson M, Bartkova S, Scherman K, Strandh M, et al. Evolution of antigenic diversity in the ticktransmitted bacterium Borrelia afzelii: a role for host specialization? J Evol Biol. 2017;30:1034-41.

31. Pérez D, Kneubühler Y, Rais O, Jouda F, Gern L. Borrelia afzelii $o s p C$ genotype diversity in Ixodes ricinus questing ticks and ticks from rodents in two Lyme borreliosis endemic areas: Contribution of co-feeding ticks. Ticks Tick Borne Dis. 2011;2:137-42.
32. Rynkiewicz EC, Brown J, Tufts DM, Huang C-I, Kampen H, Bent $\mathrm{SJ}$, et al. Closely-related Borrelia burgdorferi (sensu stricto) strains exhibit similar fitness in single infections and asymmetric competition in multiple infections. Parasit Vectors. 2017;10:1-9.

33. Belli A, Sarr A, Rais O, Rego ROM, Voordouw MJ. Ticks infected via co-feeding transmission can transmit Lyme borreliosis to vertebrate hosts. Sci Rep. 2017;7:1-13.

34. Jacquet M, Margos G, Fingerle V, Voordouw MJ. Comparison of the lifetime host-to-tick transmission between two strains of the Lyme disease pathogen Borrelia afzelii. Parasit Vectors 2016; 9:1-8.

35. Tonetti N, Voordouw MJ, Durand J, Monnier S, Gern L. Genetic variation in transmission success of the Lyme borreliosis pathogen Borrelia afzelii. Ticks Tick Borne Dis. 2015;6:334-43.

36. Gomez-Chamorro A, Battilotti F, Cayol C, Mappes T, Koskela E, Boulanger N, et al. Susceptibility to infection with Borrelia afzelii and TLR2 polymorphism in a wild reservoir host. Sci Rep. 2019;9:1-12.

37. Gomez-Chamorro A, Heinrich V, Sarr A, Roethlisberger O, Genné D, Bregnard C, et al. Maternal antibodies provide bank voles with strain-specific protection against infection by the Lyme disease pathogen. Appl Environ Microbiol. 2019;85:1-12.

38. Baum E, Hue F, Barbour AG. Experimental infections of the reservoir species Peromyscus leucopus with diverse strains of Borrelia burgdorferi, a Lyme disease agent. mBio. 2012;3:1-11.

39. Zhong X, Nouri M, Råberg L. Colonization and pathology of Borrelia afzelii in its natural hosts. Ticks Tick Borne Dis. 2019;10:822-7.

40. Wang G, Ojaimi C, Iyer R, Saksenberg V, McClain SA, Wormser $\mathrm{GP}$, et al. Impact of genotypic variation of Borrelia burgdorferi sensu stricto on kinetics of dissemination and severity of disease in C3H/HeJ mice. Infect Immun. 2001;69:4303-12.

41. Wang GQ, Ojaimi C, Wu HY, Saksenberg V, Iyer R, Liveris D, et al. Disease severity in a murine model of Lyme borreliosis is associated with the genotype of the infecting Borrelia burgdorferi sensu stricto strain. J Infect Dis. 2002;186:782-91.

42. de Roode JC, Helinski MEH, Anwar MA, Read AF. Dynamics of multiple infection and within-host competition in genetically diverse malaria infections. Am Nat. 2005;166:531-42.

43. Derdakova M, Dudioak V, Brei B, Brownstein JS, Schwartz I, Fish D. Interaction and transmission of two Borrelia burgdorferi sensu stricto strains in a tick-rodent maintenance system. Appl Environ Microbiol. 2004;70:6783-8.

44. Hibbing ME, Fuqua C, Parsek MR, Peterson SB. Bacterial competition: surviving and thriving in the microbial jungle. Nat Rev Microbiol. 2009;8:15-25.

45. Wale N, Sim DG, Read AF. A nutrient mediates intraspecific competition between rodent malaria parasites in vivo. P R Soc BBiol Sci. 2017;284:1-8.

46. Mideo N, Barclay VC, Chan BHK, Savill NJ, Read AF, Day T. Understanding and predicting strain-specific patterns of pathogenesis in the rodent malaria Plasmodium chabaudi. Am Nat. 2008;172:E214-38.

47. Raberg L, de Roode JC, Bell AS, Stamou P, Gray D, Read AF. The role of immune-mediated apparent competition in genetically diverse malaria infections. Am Nat. 2006;168:41-53.

48. Fairlie-Clarke KJ, Allen JE, Read AF, Graham AL. Quantifying variation in the potential for antibody-mediated apparent competition among nine genotypes of the rodent malaria parasite Plasmodium chabaudi. Infect Genet Evolution. 2013;20:270-5.

49. Tilly K, Rosa PA, Stewart PE. Biology of infection with Borrelia burgdorferi. Infect Dis Clin North Am. 2008;22:217-34.

50. Hartemink NA, Randolph SE, Davis SA, Heesterbeek JAP. The basic reproduction number for complex disease systems: Defining R-0 for tick-borne infections. Am Nat. 2008;171:743-54. 
51. Mackinnon MJ, Read AF. Genetic relationships between parasite virulence and transmission in the rodent malaria Plasmodium chabaudi. Evolution. 1999;53:689-703.

52. Mackinnon MJ, Read AF. The effects of host immunity on virulence-transmissibility relationships in the rodent malaria parasite Plasmodium chabaudi. Parasitology. 2003;126:103-12.

53. Brisson D, Dykhuizen DE. ospC diversity in Borrelia burgdorferi: different hosts are different niches. Genetics. 2004;168:713-22.

54. Wang IN, Dykhuizen DE, Qiu W, Dunn JJ, Bosler EM, Luft BJ. Genetic diversity of $\operatorname{spC}$ in a local population of Borrelia burgdorferi sensu stricto. Genetics. 1999;151:15-30.
55. Qiu WG, Bosler EM, Campbell JR, Ugine GD, Wang IN, Luft BJ, et al. A population genetic study of Borrelia burgdorferi sensu stricto from eastern Long Island, New York, suggested frequency-dependent selection, gene flow and host adaptation. Hereditas. 1997;127:203-16.

56. Qiu WG, Dykhuizen DE, Acosta MS, Luft BJ. Geographic uniformity of the Lyme disease spirochete (Borrelia burgdorferi) and its shared history with tick vector (Ixodes scapularis) in the northeastern United States. Genetics. 2002;160:833-49.

57. Brisson D, Drecktrah D, Eggers C, Samuels DS. Genetics of Borrelia burgdorferi. Annu Rev Genet. 2012;46: 515-36. 